Canadian University Music Review

Canadian University Music Review

Revue de musique des universités canadiennes

\title{
Richard Strauss à l'oeuvre : les premières esquisses pour Salome
}

Jean-Michel Boulay

Volume 17, numéro 2, 1997

URI : https://id.erudit.org/iderudit/1014786ar

DOI : https://doi.org/10.7202/1014786ar

Aller au sommaire du numéro

\section{Éditeur(s)}

Canadian University Music Society / Société de musique des universités canadiennes

\section{ISSN}

0710-0353 (imprimé)

2291-2436 (numérique)

Découvrir la revue

Citer cet article

Boulay, J.-M. (1997). Richard Strauss à l'oeuvre : les premières esquisses pour Salome. Canadian University Music Review / Revue de musique des universités canadiennes, 17(2), 49-70. https://doi.org/10.7202/1014786ar
Résumé de l'article

L'auteur, après avoir tenté d'établir à quel moment Richard Strauss commence son travail sur Salome, fait un inventaire des sources manuscrites de l'opéra. Puis il se concentre sur un exemplaire de la première édition de la traduction allemande de la pièce d'Oscar Wilde ayant appartenu au compositeur et qui contient de nombreuses annotations musicales. Une classification et une description de celles-ci sont suivies de l'analyse détaillée du premier stade de conception de deux passages clés de Salome.
All Rights Reserved ( C Canadian University Music Society / Société de musique des universités canadiennes, 1997
Ce document est protégé par la loi sur le droit d'auteur. L'utilisation des services d'Érudit (y compris la reproduction) est assujettie à sa politique d'utilisation que vous pouvez consulter en ligne.

https://apropos.erudit.org/fr/usagers/politique-dutilisation/ 


\title{
RICHARD STRAUSS À L'GEUVRE : LES PREMIÈRES ESQUISSES POUR SALOME
}

\author{
Jean-Michel Boulay
}

La création à Dresde, le 9 décembre 1905, de la Salome de Richard Strauss a sans aucun doute constitué l'événement marquant de cette saison musicale allemande. En effet, on peut sans crainte affirmer que le monde musical germanique dans son entier en a subi les contrecoups. De nombreux musiciens, et non des moindres, ont émis l'opinion que la musique, après cette nouvelle œuvre de Strauss, ne pourrait plus jamais rester la même et que tous les compositeurs se trouveraient forcés de réagir, favorablement ou non, à cette nouvelle direction créatrice. De plus, l'émoi causé par Salome n'a pas été exclusivement l'affaire de musiciens, le grand public y ayant pris lui aussi une part active. Ainsi, la plupart des journaux et des revues culturelles et musicales ont consacré de nombreuses pages aux articles, aux recensions et aux lettres des lecteurs sur le nouvel opéra. Certaines réactions ont même été plutôt extrêmes : on a publié des pamphlets accusant Strauss d'encourager la dépravation des mœurs et l'anarchie musicale ${ }^{1}$, on a censuré certaines parties essentielles de l'œuvre $^{2}$ et des fanatiques ont même organisé des manifestations publiques contre celle-ci.

C'est sur les compositeurs d'avant-garde que l'impact de Salome s'est particulièrement fait sentir. Arnold Schönberg, par exemple, a maintes fois déclaré que Strauss avait écrit là un chef-d'œuvre digne d'une étude attentive ${ }^{3}$. Anton Webern, qui est toujours resté critique vis-à-vis de Strauss, admettait pourtant ouvertement que Salome était un chef-d'œuvre ${ }^{4}$. Alban Berg, quant à lui, avait une admiration profonde pour Salome et une excellente connaissance de l'œuvre, qu'il essayait d'entendre le plus souvent possible, même si cela pouvait exiger des déplacements importants ${ }^{5}$. On peut d'ailleurs se demander

1 Voir, par exemple, le pamphlet de H. Ernstman, Salome an den deutschen Hofbühnen : ein Kulturbild (Berlin : H. Walter, 1906). Pour une interprétation particulièrement intéressante de ces réactions, on pourra consulter Sander L. Gilman, « Strauss and the Pervert », dans Reading Opera, édité par Arthur Gross et Roger Parker (Princeton : Princeton University Press, 1989), 306-27.

2 Par exemple, Salome a été interdite à Vienne jusqu'en 1918 et la première production berlinoise, donnée le 5 décembre 1906, contenait de multiples révisions obligées et une réinterprétation religieuse de la scène finale.

3Willi Reich, Schoenberg : A Critical Biography (New York : Da Capo Press, 1981), 25. Pour en savoir plus sur les rapports entre Strauss et Schönberg, on consultera le troisième chapitre, « Schoenberg et Strauss », de Hans Heinz Stuckenschmidt, Arnold Schoenberg, traduit par Hans Hildenbrand (Paris : Fayard, 1993), 65-82.

4Friedrich Deutsch-Dorian, «Webern als Lehrer », Melos 27, $n^{\circ} 4$ (avril 1960) : 104.

5II semble d'ailleurs que Berg se soit intéressé à la musique de Strauss pendant assez longtemps. Par exemple, dans le deuxième acte de Wozzeck (mes. 603 et suivantes), Berg fait clairement allusion 
comment Wozzeck et Lulu auraient pu être composés sans l'influence de Salome $e^{6}$. Quant à Strauss lui-même, c'est dans cet opéra qu'il a réalisé sa première grande synthèse entre la tonalité chromatique et les techniques les plus avancées de manipulation motivique, un amalgame qui a abouti, on le sait, au chromatisme radical d'Elektra? .

Dans le texte suivant, j'essaierai d'analyser la première étape du processus de création de Salome. Cette étude, essentielle à une meilleure compréhension de cette musique complexe, se fera à partir du livret annoté de l'opéra, un document particulièrement riche en indices de toutes sortes. Après avoir tenté de déterminer aussi précisément que possible la date du début du travail compositionnel et après avoir fait un rapide survol de la bibliographie, je me pencherai sur le livret annoté lui-même. Je le décrira en détail et tenterai d'en expliquer le rôle dans l'élaboration de l'opéra. On pourra donc observer l'émergence des idées musicales dans l'esprit de Strauss et identifier les caractéristiques qui sont les plus importantes à ses yeux. Ces observations permettront non seulement de mieux comprendre comment Salome a pu voir le jour, mais aussi de faire le tri entre les éléments essentiels de la musique et les détails de moindre importance.

\section{Début du travail de composition}

Strauss a lui-même déclaré avoir commencé son travail sur Salome vers la fin de 1902 ou au début de 1903, c'est-à-dire à l'époque où il travaillait à la mélodie avec orchestre Das Thal, op. 51, $\mathrm{n}^{\circ} 1$, à la ballade Taillefer, op. 52, et à la Symphonia domestica, op. 53. Dans sa biographie de Strauss, Norman Del Mar écrit que le compositeur a vu la Salomé d'Oscar Wilde pour la première fois au début de 1903, dans une production en allemand du Kleines Theater de Berlin, sous la direction de Max Reinhardt et avec Gertrud Eysoldt dans le rôle titre $^{8}$. Mais il ne semble pas que ce soit cette représentation qui ait éveillé l'intérêt du compositeur pour l'histoire de la fille d'Hérodias, mais bien une proposition de collaboration du poète viennois Anton Lindner (1874-1915),

à une valse du deuxième acte de Rosenkavalier. Cette allusion est relevée dans George Perle, The Operas of Alban Berg (Berkeley: University of California Press, 1980), vol. 1, Wozzeck, 74.

6Derrick Puffett montre l'influence de Salome sur un passage de Wozzeck dans son article « Berg and German Opera ", dans The Berg Companion, édité par Douglas Jarman (Londres : Macmillan, 1989), 200-203. Dans cet article, Puffett relève aussi des emprunts directs, comme celui de la «lune rouge sang " de l'acte III de l'opéra de Berg.

7 Pour en savoir plus sur les aspects théoriques et techniques du langage musical de Salome, on pourra consulter les ouvrages suivants : Tethys Carpenter, "Tonal and Dramatic Structure ", dans Richard Strauss : Salome, édité par Derrick Puffett (Cambridge : Cambridge University Press, 1989), 88-108; Norman H. Dinerstein, « Polychordality in Salome and Elektra : A Study of the Reinterpretation Technique » (thèse de doctorat, Princeton University, 1984); Edward Murphy, «Tonality and Form in Salome ", The Music Review 50, $\mathrm{n}^{\text {os }} 3 / 4$ (aout-novembre 1989) : 215-30; Jean-Michel Boulay, « Monotonality and Chromatic Dualism in Richard Strauss's Salome » (thèse de doctorat, University of British Columbia, 1992).

8 « [...] early in 1903 [Strauss] took the opportunity of the Berlin production to go and see the play for himself, drawn additionally by the appearance of the prominent actress Gertrud Eysoldt in the title role. » Norman Del Mar, Richard Strauss : A Critical Commentary on His Life and Works (Ithaca : Cornell University Press, 1986), 1:243. La creation allemande de la pièce de Wilde a eu lieu à Breslau en 1901. 
dont Strauss avait mis en musique un poème, le Hochzeitlich Lied, en 1898 (op. $\left.37, \mathrm{n}^{\circ} 6\right)^{9}$. Lindner voulait écrire un livret en vers à partir de la pièce de Wilde. Dans ses Betrachtungen und Erinnerungen, Strauss raconte avoir reçu et rejeté l'offre de Lindner et avoir décidé de travailler directement à partir de la traduction allemande de la pièce de Wilde par Hedwig Lachmann, et ce avant d'avoir assisté à la représentation berlinoise.

J'étais un jour à Berlin, dans le «Thêatre de poche » de Max Reinhardt, pour voir Gertrude Eysoldt dans le rôle de Salomé de Wilde. Après la représentation je fis la rencontre d'Henri Grünfeld qui me dit : « Strauss, voilà un sujet d'opéra pour vous ». Je répondis : « J'y travaille ». Le poète lyrique viennois Antoine Lindner m'avait déjà envoyé ce texte délicieux en me proposant de le transformer en livret d'opéra. J'avais acquiescé et il m'avait remis par la suite quelques premières scènes versifiées avec beaucoup d'habileté sans que je pusse me décider à les mettre en musique, jusqu'au jour où il me vint à l'esprit de composer un air sur ce motif personnel : «Qu'elle est belle, cette nuit, la princesse Salomé! » À partir de ce moment, il ne me fut pas difficile de purger le poème de la littérature qu'il contenait et d'en faire un très beau «livret $»^{10}$.

Ce texte nous permet de dater de façon assez précise le début du travail sur Salome. En effet, Reinhardt a présenté la pièce de Wilde pour la première fois le 15 novembre 1902 devant un parterre d'invités. Une deuxième représentation, réservée aux membres de la Lessing-Gesellschaft, a eu lieu le 22 février $1903^{11}$. Si l'on en croit Del Mar, et compte tenu du scénario évoqué par Strauss, ce dernier aurait commencé son travail au plus tard quelques semaines avant la fin de février 1903, plus vraisemblablement à la toute fin de 1902. C'est un scénario possible, mais qui semble être contredit par les sources manuscrites de l'opéra ${ }^{12}$. Une deuxième hypothèse serait que Strauss ait assisté à l'une des premières représentations de la deuxième production de Salomé (102 représentations en un an), c'est-à-dire entre le 26 septembre et le 8 octobre 1903, car Gertrud Eysoldt ne semble avoir participé qu'aux représentations données entre ces deux dates ${ }^{13}$. Ceci voudrait dire que Strauss aurait commencé ses ébauches autour de juillet 1903.

\section{Sources documentaires}

Le seul véritable catalogue des esquisses de Strauss a été établi en 1977 par Franz Trenner ${ }^{14}$. Il s'agit d'un essai d'inventaire des 144 livrets d'esquisses conservés aux archives Strauss de Garmisch et dont on peut trouver copie sur

\footnotetext{
9 Ce lied est surtout connu pour sa citation exacte du motif de Vénus de Tannhäuser.

10 Richard Strauss, Anecdotes et souvenirs, version française de Pierre Meylan et Jean Schneider (Lausanne : Éditions du Cervin, 1951), 42.

11 Heinrich Huesmann, Welttheater Reinhardt : Bauten, Spielstätten, Inszenierungen (Munich : Prestel, 1983), annexe « Inszenierungen », entré 167.

12Le carnet d'esquisse numéro 11, conservé aux archives Strauss de Garmisch, porte la mention « Begonnen am 27. Juli 1903 » (fol. 1v).

13Huesmann, annexe * Inszenierungen », entrée 204.

14Franz Trenner, Die Skizzenbücher von Richard Strauss aus dem Richard-Strauss-Archiv in Garmisch (Tutzing : Hans Schneider, 1977).
} 
microfilm à la Bayerische Staatsbibliothek de Munich ${ }^{15}$. Ce catalogue de Trenner, un outil indispensable à toute recherche sur Strauss, est malheureusement peu précis dans ses descriptions du contenu détaillé des livrets. Il existe très peu d'inventaires détaillés de ces livrets d'esquisses, et aucun inventaire des carnets qui contiennent du matériel pour Salome ${ }^{16}$. On peut parfois compléter les renseignements donnés par Trenner dans son catalogue d'esquisses en consultant son catalogue général des œuvres de Strauss ainsi que le catalogue thématique de Mueller von Asow ${ }^{17}$. Jusqu'à maintenant, les musicologues intéressés par le processus de composition chez Strauss se sont surtout penchés sur Elektra, Daphne, les Metamorphosen et les Vier letzte Lieder ${ }^{18}$. Le seul texte ayant trait au processus de composition de Salome est une courte étude du livret annoté, réalisée par Roland Tenschert en $1960^{19}$.

Comme pour les autres opéras de Strauss, les sources manuscrites de Salome peuvent être classées en quatre catégories correspondant à quatre étapes de composition : livret annoté, esquisses, particelle, partition d'orchestre. On a souvent commenté l'habitude qu'avait Strauss d'annoter musicalement son exemplaire du livret ${ }^{20}$. Ces annotations consistent en général en indications harmoniques ou tonales et parfois en de courtes notations de motifs ou de formules rythmiques. C'est donc dans le livret que Strauss avait l'habitude de noter ses premières impressions musicales. D'après Gilliam, les esquisses, contenues dans de petits calepins, peuvent être divisées en esquisses préliminaires et en esquisses finales, écrites au crayon ${ }^{21}$. La particelle est d'habitude faite à l'encre, comme d'ailleurs la partition d'orchestre finale. Nous nous concentrerons ici sur la première étape, celle du livret annoté.

15Bayerische Staatsbibliothek, cote Mus. fm 1 \# 1-144. Ces microfilms sont la propriété de la famille Strauss. L'auteur aimerait remercier le Dr. Richard Strauss, petit-fils du compositeur, d'avoir eu l'amabilité de lui donner la permission de copier une très grande partie des sources mentionnées dans cet article.

16Les deux exceptions principales sont dues à Bryan Gilliam. On pourra consulter, pour Elektra, son livre Richard Strauss's « Elektra * (Oxford : Oxford University Press, 1991) et, pour Daphne, sa thèse " Richard Strauss's Daphne : Opera and Symphonic Continuity » (thèse de doctorat, Harvard University, 1984).

17Erich H. Mueller von Asow, Richard Strauss : thematisches Verzeichnis, 3 vol. (Vienne : Doblinger, 1959), et Franz Trenner, Richard Strauss : Werkverzeichnis (Vienne : Doblinger, 1985).

18Pour Elektra, on consultera le livre de Bryan Gilliam mentionné plus haut, mais aussi son article «Strauss's Preliminary Opera Sketches : Thematic Fragments and Symphonic Continuity », 19th-Century Music, 9, $\mathrm{n}^{\circ} 3$ (1986) : 176-88. Pour Daphne, voir la thèse de Gilliam déjà mentionnée. Pour les Metamorphosen, voir Timothy L. Jackson, « The Metamorphosis of the Metamorphosen : New Analytical and Source-Critical Discoveries ", dans Richard Strauss : New Perspectives on the Composer and His Work, édité par Bryan Gilliam (Durham : Duke University Press, 1992), 193-241. Pour les Vier letzte Lieder, voir la thèse de Timothy L. Jackson, "The Last Strauss : Studies of the Letzte Lieder " (Graduate Center of the City University of New York, 1988) et, du même auteur, « Ruhe, meine Seele! and the Letzte Orchesterlieder ", dans Richard Strauss and His World, édite par Bryan Gilliam (Princeton : Princeton University Press, 1992), 90-137.

19Roland Tenschert, "Richard Strauss's Opernfassung der deutschen Übersetzung von Oscar Wildes Salome ", Richard-Strauss-Jahrbuch (1959-60) : 99-106. Ce texte a été traduit en anglais et augmenté de quelques commentaires de Derrick Puffett dans Richard Strauss : « Salome », 36-50.

20Voir, en plus des sources déjà mentionnées, Charlotte Erwin, "Richard Strauss's Presketch Planning of Ariadne auf Naxos », The Musical Quarterly 67, $\mathrm{n}^{\circ} 3$ (juillet 1981) : 348-65.

21 Bryan Gillian, Richard Strauss's * Elektra * (Oxford : Oxford University Press, 1991), 109-10. 


\section{Le livret annoté}

Le livret annoté de Salome est un exemplaire de la première édition de la traduction allemande de la pièce d'Oscar Wilde, réalisée par Hedwig Lachmann et publiée à Leipzig en 1903 par les éditions Insel22. Cet exemplaire est aujourd'hui conservé aux archives Strauss de Garmisch. La traduction de Lachmann est la plus connue et la plus utilisée des traductions allemandes de l'œuvre ${ }^{23}$. En 1903, Hedwig Lachmann (1865-1918) était déjà bien connue comme traductrice et poétesse. Née à Storp, en Poméranie, et fille du professeur de religion juive Isaak Lachmann (1838-1900), elle s'intéresse très tôt aux langues étrangères. Elle travaille d'abord comme enseignante en Angleterre, à Dresde, à Budapest et à Berlin, où elle se lie d'amitié avec le poète Richard Dehmel. À partir de 1891, elle publie des poèmes dans des revues littéraires. La même année paraissent ses Ungarische Gedichte. Les années suivantes, elle traduit Edgar Allan Poe, Dante Gabriel Rossetti, Algernon Charles Swinburne et Paul Verlaine. Après 1903, elle participe activement aux éditions allemandes des œuvres complètes de Poe et d'Honoré de Balzac et traduit également L'homme et son âme devant la société et Le portrait de Dorian Gray de Wilde ${ }^{24}$.

La première édition de 1903 n'est pas paginée et le texte y est précédé de 10 superbes illustrations de Marcus Behmer (1879-1958) ${ }^{25}$. Behmer est l'un des plus importants illustrateurs allemands du début du siècle. Fils de Hermann Behmer, un peintre connu surtout pour ses peintures historiques, il a illustré de façon somptueuse de nombreux ouvrages des éditions Insel. Il a également collaboré à de nombreuses revues, dont Simplizissimus, Insel et Ver sacrum. Ses dessins, qui font très souvent appel au grotesque, sont influencés par l'art persan, par l'ornementation irlandaise ancienne et par le mouvement esthétiste, tout particulièrement par Aubrey Beardsley, qui avait d'ailleurs produit les illustrations pour la première édition anglaise de Salomé. Ce sont les illustrations de Behmer pour la pièce de Wilde qui lui ont assuré sa renommée. Celle-ci a été par la suite confirmée par ses illustrations pour le Westöstlicher Divan de Goethe, pour les contes de Grimm et pour Zaïre de Voltaire.

Sur la page de garde, Strauss dresse la liste des personnages, qui correspond en tous points à celle de l'opéra. Après les illustrations déjà mentionnées, on retrouve 56 pages de texte, entrecoupées de 9 pages d'illustrations. À première vue, Strauss semble avoir paginé lui-même son exemplaire. En effet, on retrouve dans la marge extérieure un numéro de page entre crochets. Or,

22Le fait que la première édition de la traduction de Lachmann a été publiée en 1903 est un argument de plus pour la date proposée plus haut pour le début du travail sur l'opéra.

23 Une autre traduction a été réalisée par Isidore Leo Pavia et Hermann Freiherr von Teschenberg. Elle a elle aussi été publiée pour la première fois en 1903 à Leipzig, mais aux éditions Max Spohr. Une troisième traduction a été faite en 1918 par Paul Stegemann. Elle a été publiée la même année, à Hannovre, aux éditions Heinrich Böhme.

24Ulrich Karthaus, « Nachwort », dans Oscar Wilde, Salome : Tragödie in einem Akt (Stuttgart : Reclam, 1990), 65-66.

25Les éditions subséquentes, paginées celles-là et complètement recomposées, ne comporteront que deux pages d'illustrations suivies du texte. L'ordre des illustrations à l'intérieur du texte sera d'ailleurs complètement différent. Par contre, la première page de texte portera le numéro de page 11. 
premièrement, Strauss donne un numéro impair, 13 , à la deuxième page de texte, même s'il s'agit d'une page de gauche. Deuxièmement, il manque une page 21. Troisièmement, les pages d'illustrations à l'intérieur du texte ne sont pas numérotées. Quatrièmement, certains des numéros sont accompagnés d'un trait qui les relie à un interligne situé à un endroit bien précis de la page. Enfin, les numéros 34 et 35 se retrouvent sur la même page. La comparaison avec un exemplaire d'une édition subséquente permet de résoudre le problème : ces numéros de pages entre crochets correspondent exactement à ceux des éditions subséquentes. On est donc en droit de se demander si une deuxième copie du livret (peut-être annotée elle aussi), aujourd'hui perdue, a existé et que Strauss avait inventé ce système de pagination pour coordonner son travail. Il se peut aussi, et c'est là une hypothèse encore plus probable, que Strauss ait utilisé ces numéros de pages pour correspondre avec quelqu'un au sujet de son travail. Nous utiliserons ici une pagination par folio, avec pour première page de texte le fol. $1 \mathrm{r}$.

On retrouve dans ce livret 10 catégories d'annotations manuscrites : (1) coupures; (2) transformations du texte; (3) soulignements; (4) divisions formelles; (5) indications de tonalités; (6) indications d'accords ou de noms de notes; (7) indications de mesures; (8) numéros de mesures; (9) indications textuelles pour la composition; (10) notations musicales.

Les coupures peuvent prendre trois formes : soit des traits horizontaux recouvrant le texte coupé, soit de longs traits diagonaux simples ou multiples et dont les extrémités sont la plupart du temps marquées par des « +» (exemple 1), soit une combinaison de longs traits diagonaux et de traits verticaux en marge, accompagnés de l'annotation "Strich ». Les tranformations du texte existant accompagnent en général une coupure et sont placées en marge (exemple 2), mais elles peuvent aussi être insérées à l'intérieur d'une phrase. Le soulignement est employé pour faire ressortir certains mots clés, surtout lorsque ceux-ci correspondent à des annotations à caractère musical placées en marge. Les désignations de tonalités ou d'accords sont placées soit au-dessus ou au-dessous du texte, soit en marge, alors que des numéros de mesure sont parfois inscrits au-dessus du texte, de façon à mieux en marquer le rythme. Les indications de mesure, les indications relatives à la composition des détails de surface $^{26}$ et les notations musicales sont toutes placées en marge.

Le rôle des coupures est particulièrement important et mérite d'être analysé en détail. L'emploi de motifs textuels est l'un des aspects les plus marquants de la Salomé d'Oscar Wilde. Des mots, des expressions, des phrases ou des images y sont répétées de manière incantatoire, dans le but, semble-t-il, de créer une atmosphère hypnotique. Cette technique de répétition semble avoir été utilisée de façon délibérée et non à cause d'une maîtrise imparfaite de la langue française, comme l'ont laissé croire certains commentateurs anglophones ${ }^{27}$. En fait, plusieurs écrivains français importants ont admiré Salomé. Nous ne citerons ici que Stéphane Mallarmé, qui écrit dans une lettre de mars 1893 :

26Le terme « surface » est employé ici dans sons sens schenkérien d'avant-plan structurel.

27 Je pense ici particulièrement à Arthur Ransome, Oscar Wilde : A Critical Study (New York : Haskell House, 1971 [réimpression de l'édition de 1912]), et à Christopher S. Nassaar, Into the Demon Universe : A Literary Exploration of Oscar Wilde (New Haven : Yale University Press, 1974). 
ERSTER SOLDAT: Er hat sich selbst getötet, Herr. HERODES: Auswetehem Grund? Teh hatteitm zum Hauptmanmeiner Leibwache ernannt! ZWEITER SOLDAT: Wir wissen es nich, Herr. Abermiteigener Hand hat er sieh getötet. HERODES: Das scheint mir seltsam. Ich hätte gedacht, nur die römischen Philosophan töten sich selbst. Nicht wahr, Tigellinus, die Phlosophen in Rom töten sich selbst? TIGELLINUS: Es gibt dort einige, die sich seybst töten. Es sind die Stoiker. Die Stoiker sind Leute ohne Bildung. Es sind lächerliche Leute. Ich für meinen Theil halte sie für ganz und gar lächerlich.

Exemple 1 : Livret annoté, bas du fol. 14v.

DER PAGE DER HERODIAS: Sieh sie nicht an. Ich bitte dich, sieh sie nicht an. DER JUNGE SYRIER: Sie ist wie cine Taube, die Sie ist sich verirrthat... Sie istwie eine Narcisse, die wie eine im-Winde zittert...Sie ist wie eine silberne Btume. verirte SALOME tritt ein. Taube.

Exemple 2 : Livret annoté, bas du fol. 3v.

J'admire que tout étant exprimé par de perpétuels traits éblouissants, en votre Salomé, il se dégage, aussi, à chaque page, de l'indicible et le Songe. Ainsi les gemmes innombrables et exactes ne peuvent servir que d'accompagnement sur sa robe au geste surnaturel de cette jeune princesse, que définitivement vous évoquâtes ${ }^{28}$.

Wilde lui-même confirmé ses intentions dans une lettre à Alfred Douglas, le traducteur anglais de Salomé : "Les phrases récurrentes de Salomé, qui l'unissent ainsi que les motifs une œuvre musicale, sont, et étaient pour moi, l'équivalent artistique des refrains des vieilles ballades ${ }^{29}$."

28Cité dans Richard Ellmann, Oscar Wilde (New York : Knopf, 1987), 354. On trouvera au même endroit d'autres témoignages de Pierre Loti et de Maurice Maeterlinck.

29 « The recurring phrases of Salome, that bind it together like a piece of music with recurring motifs, are, and were to me, the artistic equivalent of the refrains of old ballads. " Cité dans Norbert Kohl, Oscar Wilde : das literarische Werk zwischen Provokation und Anpassung (Heidelberg : Carl Winter, 1980), 304. 
Par ses coupures, Strauss cherche à mettre en valeur les motifs textuels de Wilde. Il retranche les répétitions immédiates pour ne garder que celles qui établissent des liens structurels à plus long terme. Par exemple, la phrase "Schreckliches kann geschehen " $\left(8^{1-3}\right)$ apparaît au tout début de l'opéra et est alors associée aux craintes du page d'Hérodias ${ }^{30}$. Elle est ensuite transformée en « Schreckliches wird geschehen » $\left(28^{4-7}\right)$, puis en " Ich weiss, es wird Schreckliches geschehen » (48 $\left.{ }^{9-11}\right)$. Le motif réapparaît dans la deuxième partie de l'opéra, dans la bouche d'Hérode, sous les formes " gewiss wird Unheil über einen kommen » $\left(303^{3-4}\right)$ et « es wird Unheil geschehen » $\left(304^{2-3}\right)$.

Certaines répétitions sont beaucoup plus complexes. Ce qu'on pourrait appeler le « motif du regard » est omniprésent dans l'opéra et apparaît à des moments essentiels. Au début, c'est Narraboth qui regarde Salomé, tandis que le page lui demande de cesser de la regarder ainsi. Lorsque Salomé entre en scène, elle se plaint du fait que Hérode la regarde continuellement « mit seinen Maulwurfsaugen unter den zuckenden Lidern » $\left(22^{4-8}\right)$. Puis, lorsqu'elle tente de convaincre Narraboth de la laisser voir Jochanaan, elle lui promet de le regarder le lendemain matin : "Morgen früh werde ich unter den Muss'linschleiern dir einen Blick zuwerfen. Narraboth, ich werde dich ansehn, kann sein, ich werde dir zulächeln. Sieh mich an, Narraboth, sieh mich an » (561$57^{6}$ ). Jochanaan accuse Herodias de s'être laissée emporter à la concupiscence de ses yeux : "Wo ist sie, die sich hingab der Lust ihrer Augen » $\left(69^{5}-70^{1}\right)$. Le premier commentaire de Salomé sur l'apparence physique de Jochanaan concerne ses yeux, qui sont " wie die schwarzen Höhlen, wo die Drachen hausen » $\left(77^{1-3}\right)$. Pour sa part, le prophète, lorsqu'il prend conscience pour la première fois de la présence de Salomé, parle de ses yeux en variant l'image déjà mentionnée : "ihren Goldaugen unter den gleissenden Lidern 》 $\left(81^{9}\right.$ $82^{1}$ ). Quant à Narraboth, juste avant son suicide, il implore Salomé de ne pas regarder le prophète : " sieh diesen Mann nicht an " (1241-2). Finalement, lorsque Jochanaan redescend dans la citerne, il dit à Salomé : "Ich will dich nicht ansehn » (1391-2). Dans la deuxième partie de l'opéra, c'est Hérode qui regarde Salomé alors que Herodias tente de l'en empêcher. Lorsque Hérode glisse sur le sang de Narraboth et voit son cadavre, il dit : « Ich will ihn nicht sehn $\gg\left(161^{8-9}\right)$. Plus tard, les cinq juifs débattent de la possibilité que Jochanaan ait vraiment vu Dieu. Puis, dans le grand monologue de Salomé, on retrouve cette image magnifique : « wenn ich dich ansah, hörte ich geheimnisvolle Musik » (338 $\left.{ }^{3-9}\right)$. D' ailleurs, le monologue se termine sur : « Hättest du mich angesehn, du hättest mich geliebt » $\left(347^{6}-48^{2}\right)$.

Bien que le motif du regard soit omniprésent dans l'opéra, la pièce de Wilde y fait encore plus allusion. Or, tous les passages coupés qui utilisent ce thème sont secondaires à l'action principale. Nous ne citerons qu'un exemple. Entre le « $\mathrm{Ja}$, er sieht finster drein » du deuxième soldat $\left(8^{8}\right)$ et le « Auf wen blickt

30Tout au long de cette étude, on utilisera le système de numérotation de mesure suivant : la première mesure du chiffre 2, c'est-à-dire celle dont la barre de mesure de gauche est surmonté du chiffre 2 , sera noté $2^{1}$ et la mesure suivante sera noté $2^{2}$. 
er? » du premier soldat $\left(8^{9}\right)$, le texte de Wilde contient les deux répliques suivantes :

Premier soldat : Il regarde quelque chose.

Second soldat : Il regarde quelqu'un.

Il semble que, pour Strauss, ces deux répliques soulignent prématurément l'attirance de Hérode pour Salomé. Le compositeur évite ainsi de dissiper l'ambiguïté.

Un autre rôle des coupures est de clarifier l'action en omettant tous les petits détails inutiles que peut receler la pièce de Wilde. En effet, cette dernière est particulièrement riche en détours de toutes sortes, en explications et en descriptions qui n'ont pas leur place à l'opéra. L'exemple 3 reproduit une réplique de Hérode tirée du fol. 29r. Ici, le roi tente de convaincre Salomé de revenir sur sa demande en lui disant que la tête d'un homme coupée du reste de son corps est une vision horrible. Puis, il lui offre une émeraude. La première phrase coupée dit fondamentalement la même chose que la phrase suivante. La deuxième coupure (lignes 5-8) comprend deux phrases qui, elles aussi, peuvent être considérées comme redondantes et pas assez directes : " C'est quelque chose d'horrible, d'épouvantable, que ce que tu me demandes. Au fond, je crois que tu veux plaisanter. " La phrase suivante, celle que conserve Strauss, est beaucoup plus précise : "La tête d'un homme décapité, c'est une chose laide, n'est-ce-pas? » La troisième coupure (lignes 9-13) n'est qu'un commentaire sur ce qui a déjà été dit. La dernière coupure, pour sa part, est la plus longue. Ici, Hérode décrit son émeraude : elle est ronde, l'amie de César la lui a envoyée, elle permet de voir au loin; César en possède une semblable, mais plus petite. Strauss va directement à l'essentiel : «C'est la plus grande émeraude du monde. » On remarquera également que les coupures de clarification permettent aussi à Strauss d'éliminer quatre personnages secondaires : Tigellin, le Nubien, le Sadducéen et le Pharisien.

Un dernier rôle des coupures est de mettre en valeur la structure du livret. L'opéra, comme d'ailleurs la pièce de Wilde, est très riche en structures ternaires. Par exemple, la scène entre Salomé et Jochanaan $\left(92-122^{9}\right)$ est clairement divisée en trois parties ${ }^{31}$ :

(1) Salomé : «Ich bin verliebt in deinen Leib, Jochanaan! » Jochanaan : «Zurück, Tochter Babylons! »

Salomé : « Dein Leib ist grauenvoll. »

(2) Salomé : «In dein Haar bin ich verliebt, Jochanaan. »

Jochanaan : «Zurück, Tochter Sodoms! »

Salomé : « Dein Haar ist grässlich. »

(3) Salomé : « Deinen Mund begehre ich, Jochanaan. »

Jochanaan : « Niemals! »

31 Pour en savoir plus sur les structures ternaires dans Salome, on pourra consulter Boulay, * Monotonality and Chromatic Dualism in Richard Strauss's Salome ", 29-35. 
HERODES: Still! Sprich nicht zu mir! . ... Sa-

lome, ich beschwöre dich, sei nicht trotzig. teh bin immer zutirgewesen. Ich habe dich immer lieb gehabt.... Kann sein, ich habe dich zu lieb gehabt. Darum verlange das nicht von mir. Đas istetwas Schreektiches, etwas Gratuenvolles, was dut von-mirvertangst. Sicher, ich gtaube, du willst seherzen. Der Kopf eines Mannes, der von Rumpf getrennt ist, das ist ein übler Anblick, nicht? ziemt sich nicht, dass die-Augen eines Müdehens aufsoctwas fallen. Was für cine tust könntest du darin finden? Ou könntest keine Eust darin finden. Nein, nein, das begehrst du nieht. Horch, was ich sage. Ich habe einen Smaragd, einengrossen Smaragd, einen runden, den Cäsars Freundin mir Hergeschiskt hat. Wenn du durch diesen Smaragd siehst, kanst du sehen, was weit weg vor sich geht. Cäsar selbst trägt solch ethen Smaragd, wenn er in den Circus geht. Aber mein Smarragd ist der grössere. Ach weisses, ex - ist der grössert Er ist der grösste Smaragd in der ganzen Welt. Den willst du haben, nicht wahr? Verlange ihn von mir, ich werde ihn dir geben.

Exemple 3 : Livret annoté, haut du fol. 29r.

Certaines divisions formelles ternaires du texte sont indiquées par des lettres ou par des chiffres romains. Après l'entrée en scène de Hérode et de sa cour, on retrouve trois interventions de Herodias, dans lesquelles elle contredit ce que vient de dire Hérode : «Nein, der Mond ist wie der Mond, das ist alles » $\left(158^{7-10}\right)$, « Nein, es weht kein Wind » $\left(164^{8}-65^{1}\right)$ et « Ich höre nichts » $\left(166^{7}\right)$. Ces trois interventions sont annotées « a.) », «b.) » et «c.) ». Plus loin, Hérode offre à boire et à manger à Salomé, puis lui demande de s'asseoir sur le trône de sa mère. Les trois réponses de la princesse sont : " Ich bin nicht durstig, Tetrarch » $\left(175^{2-4}\right)$, « Ich bin nicht hungrig, Tetrarch » $\left(179^{5}-80^{1}\right)$ et « Ich bin nicht müde, Tetrarch » $\left(183^{2-4}\right)$. Ces trois répliques sont annotées «I.) », « II.) » et « III.) »

Plusieurs coupures de Strauss ont pour effet de créer de nouvelles structures ternaires à des endroits où il n'y en avait pas dans la pièce. Par exemple, dans le passage qui suit immédiatement la scène entre Salomé et Jochanaan, Salomé a, dans la pièce, sept répliques contenant les mots : «Ich will deinen Mund küssen! ». Strauss coupe la dernière de ces répliques, ce qui lui permet de regrouper les six répliques restantes en deux groupes de trois. Le premier groupe est marqué par le suicide de Narraboth, le deuxième par le sermon de Jochanaan.

Les transformations au texte conservé sont d'habitude assez légères et semblent la plupart du temps avoir été faites pour alléger certaines phrases 
complexes. Par exemple, la phrase « Er ist wie der Purpur, den die Moabiten in den Gruben von Moab finden, wie der Purpur, den die Könige, von ihnen haben » devient « Er ist wie der Purpur aus den Gruben von Moab, der Purpur der Könige ».

Les autres types d'annotations sont plus directement reliées à la musique. L'exemple 4, une transcription du fol. 1r (c'est-à-dire de la première page de texte), contient des coupures, des transformations du texte, des soulignements, des noms de tonalités, d'accords et de notes et des numéros de mesures. On remarquera d'abord les numéros de mesures : ceux-ci vont de 0 à 28 et sont la plupart du temps placés au-dessus du texte. Pour les deux premières mesures, Strauss emploie les notations « 0 . Takt » et « 1 . Takt ». Cette première division en mesures du début de l'opéra, qui a pour but de déterminer à l'avance les temps forts, est assez semblable à celle de la version finale, si on tient compte des ajustements rendus nécessaires par le fait que, dans la version finale, Narraboth commence à chanter dans la troisième mesure et non à la levée de la première mesure. Le chiffre 13, au-dessus du mot « aufsteigt » rayé, semble avoir été oublié par le compositeur après le déplacement du mot. Ce déplacement du verbe permet à Strauss de donner plus de poids grammatical et sémantique au mot « Grab », qui se retrouve ainsi en fin de phrase et sur un temps fort : "Wie eine Frau, die aufsteigt aus dem Grab ». Dans la version finale, les changements de mesure sur « kleine » (17) et « meinen » (21) sont abandonnés, vraisemblablement pour éviter un ralentissement trop prononcé de la musique, et le début de la mesure 20 est avancé d'un mot, pour mettre « Tauben » en valeur.

On peut se demander pourquoi Strauss numérote ainsi 28 barres de mesures, pour ensuite abandonner complètement ce type d'annotation. Une analyse détaillée de toutes les esquisses de Salome montrerait clairement que ce sont les points d'articulation formelle qui ont semblé donner du fil à retordre au compositeur. Chacun de ces points pose un problème compositionnel qui lui est propre. Dans ce cas, Strauss doit s'y reprendre à plusieurs fois avant de trouver une solution satisfaisante. Ici, au tout début de l'opéra, il s'agit entre autres d'établir un rythme particulier, qui mette le texte en valeur.

Cette première page contient également un grand nombre de noms de notes, d'accords et de tonalités. On voit que Strauss a dès le départ l'intention d'écrire un opéra en do dièse mineur ("Cismoll », première ligne). La musique du page doit ensuite se diriger vers des accords de la mineur (au début de la réplique du page) et de $f a$ mineur (« aussieht », souligné). On a plutôt, dans la version finale, des accords de si mineur (pour « Mondscheibe ») et de $f a$ majeur (pour « aussieht »). Harmoniquement, le premier accord n'est qu' une harmonisation passagère d'une ligne de basse conjointe allant du sol de la mesure 7 au $f a$ de la mesure $1^{3}$ (ligne sol-la-si-do dièse-ré-mi-fa), lui-même sixième degré de la mineur, sur lequel la musique cadence à la mesure $1^{7}$. Le mot souligné " seltsam » (ligne 8) renvoie à l'accord do-mi bémol-fa dièse-la, noté verticalement en marge de droite. Cet accord est bien celui que l'on retrouve dans la version finale, à la mesure $2^{2}$, mais sous la forme do-ré dièse-fa dièse-la. Ce genre de notation enharmonique est très courant dans la musique de Strauss, 


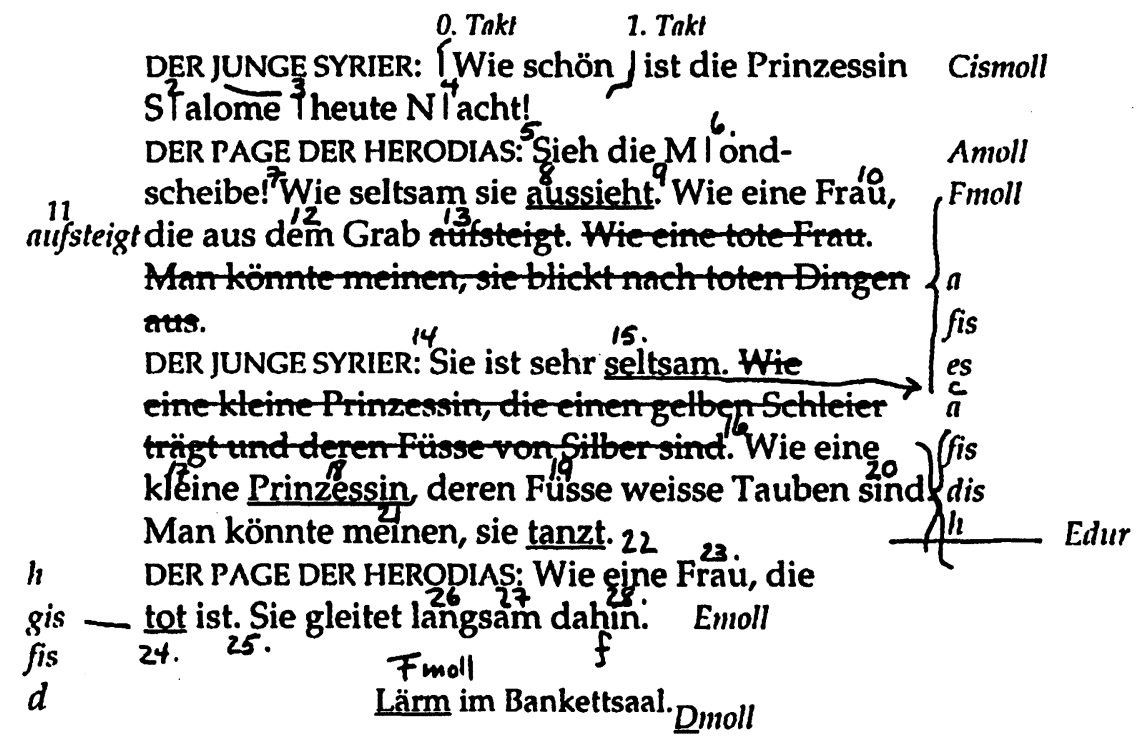

ERSTER SOLDAT: Was für ein Aufruhr! Was sind das für wilde Thiere, die da heulen? ZWEITER SOLDAT: Die Juden. Sie sind immer so. Sie streiten über ihre Religion. $\$$ Dmoll ERSTER SOLDAT: Warum streitensie tiper itmre Retigion?

Exemple 4 : Livret annoté, fol. 1r.

car le compositeur n'attachait que peu d'importance à la signification tonale de ses notations. À la ligne 11 , le soulignement de « Prinzessin » renvoie à l'accord si-ré dièse-fa dièse-la, noté en marge de droite. Cet accord est bien celui que l'on retrouve à la mesure $2^{4}$. Le mot « tanzt » souligné de la ligne suivante devait à l'origine être harmonisé par un accord de mi majeur, suivi d'un accord de mi mineur sous le mot « Frau » (ligne 13). Dans l'opéra, Strauss les remplace par des accords de do majeur-mineur (dominante de $f a, 2^{8}$ ) et de fa mineur (sus-dominante mineure de $l a, 3^{1}$ ). La musique devait ensuite se diriger vers $f a$ mineur (" dahin ») en passant par un accord ré-fa dièse-sol dièse-si (« tot »), puis, abruptement, vers ré mineur, pour accompagner le bruit venant de la salle des fêtes. Dans l'opéra, Strauss décide plutôt de faire suivre l'accord de $f a$ mineur déjà mentionné par un accord de la mineur, qui devient, par altération chromatique de la tierce, un accord de septième de dominante du ton de ré sous les mots « sie gleitet langsam ». Il n'y a plus qu'à résoudre cet accord sur sa tonique pour affirmer la nouvelle tonalité de ré mineur. 
La première page contient également trois coupures. La première (lignes 5-7) retranche deux phrases. La première phrase, " comme une femme morte », est redondante et Strauss a peut-être considéré que la deuxième, " on pourrait penser qu'elle cherche des morts ", était trop chargée de symbolisme et qu'elle vendait la mèche de façon prématurée. La deuxième coupure a probablement été faite pour éviter de surcharger le symbolisme. Ici, Wilde introduit deux couleurs secondaires, jaune et argenté, associées tout au long de la pièce au blanc de la lune/Salomé. Ce sont là des détails qui passent mal à l'opéra. La troisième coupure (deux dernières lignes) retranche des commentaires des soldats sur les motifs des querelles religieuses des juifs.

Le fol. $1 \mathrm{v}$, transcrit à l'exemple 5, est également riche en annotations musicales. On y remarque tout d'abord une courte portée en marge de gauche, contenant une idée musicale pour accompagner les mots « ist sehr schön ». Cette idée est constituée d'une levée, si bémol, suivie de trois accords : un accord de $m i$ bémol majeur, un accord de neuvième majeure sur ré et un retour à l'accord sur mi bémol. Ce passage correspond aux mesures $8^{4}$ et $8^{5}$ de la partition finale, transcrites à l'exemple 6 . On peut constater que Strauss n'a conservé que l'accord de $m i$ bémol et que celui-ci est présenté dans un contexte général de do dièse mineur, tel qu'indiqué en marge de gauche à la ligne 7.

Cette indication de tonalité renvoie aussi à la mesure $6^{3}$ (« wie schön »), qui présente la dominante de do dièse mineur. Cette dominante est suivie d'un accord enharmonique de la sixte allemande du ton de $f a$ dièse, lui-même suivi d'un 6/4 de cadence dans cette même tonalité, tel qu'indiqué à l'exemple 7. On remarquera que cet accord de $f a$ dièse majeur en deuxième renversement, qui accompagne le mot "Abend », est indiqué par Strauss à la fin de la ligne 8 du livret (exemple 5). La dernière indication musicale de cette page associe do mineur à la dernière réplique de Narraboth (ligne 20). On retrouvera cet accord de $d o$ mineur aux mesures $9^{1}$ et $9^{2}$ de la partition.

On peut dès maintenant conclure que les indications de tonalités peuvent référer soit à des régions tonales, soit à des accords, et qu'il est pratiquement impossible de faire la différence entre ces deux types d'annotations. Les noms de notes désignent en général des éléments d'un accord particulier jouant le rôle de signal, de symbole musical ${ }^{32}$.

Les indications textuelles pour la composition regroupent des annotations du type « steigend », « ostinato », etc. On peut également inclure dans cette catégorie les quelques indications de mesure que l'on retrouve parfois en marge. Par exemple, au haut du fol. $5 r$, un passage qui correspond aux mesures $35^{9}$ à $38^{8}$ de la partition finale et dont on trouvera la transcription à l'exemple 8 , Strauss planifie de marquer le contraste entre les demandes répétées de retour au banquet (2/2) et les interventions de Salomé (3/4). Cette alternance de

32Cette idée de « signal sonore » est utilisée à profusion par le musicologue Constantin Floros dans ses analyses des symphonies de Mahler, en particulier dans son ouvrage Die Symphonien, vol. 3 de Gustav Mahler (Wiesbaden : Breitkopf \& Härtel, 1985). 


ZWETER SOLDAT: Ich weiss es nicht. Sie thun
das immer. Die Pharisäer zum Beispiel sagen, dass
es Engel giabt, und die Sadducäer behaupten, dass
es keine giebt.
ERSTER SOLDAT: Ich finde es lächerlich, über
solche Dinge zu streiten.
OER JUNGE SYRIER: Wie schön ist die Prinzessin
Salome heute Abend! Fisdur
DER PAGE DER HERODIAS: Du siehst sie immer
an. Du siehst sie zuviel an. Es ist gefährlich,
Menschen auf diese Art anzusehn. Schreckliches
kann geschehen.
OER JUNGE SYRIER: Sie ist sehr schön heute Abend.
ERSTER SOLDAT: Der Tetrarch sieht finster drein.
ZWEITER SOLDAT: Ja, er sieht finster drein.
ERSTER SOLDAT: Erbticktaufetwas.
ZWEITER SOLDAT: Erbticktaufjemanden.
ERSTER SOLDAT: Auf wen blickt er?
ZWEITER SOLDAT: Ich weiss nicht.
DER JUNGE SYRIER: Wie blass die Prinzessin
ist. Niemals habe ich sie so blass gesehen. Sie
ist wie der Schatten einer weissen Rose in einem
silbernen Spiegel.
DER PAGE DER HERODIAS: Du musst sie nicht
ansehn. Du siehst sie zuviel an.
ERSTER SOLDAT: Herodias hat den Becher des geschelm
Tetrarchen gefüllt.

Exemple 5 : Livret annoté, fol. 1v.

mesures est conservée dans la version finale et le compositeur la transforme même, pour la réplique de Narraboth, en superposition.

\section{Le monologue de Salomé}

Les exemples précédents nous ont permis de déterminer quel rôle les différents types d'annotations énumérés plus haut viennent jouer dans l'élaboration des premières idées compositionnelles pour Salome. Nous terminerons cette étude par la transcription et l'analyse des annotations du monologue final de Salomé. Cette description d'une scène entière nous permettra de remettre les observations précédentes en contexte et de mieux comprendre le degré d'interaction entre différents types d'annotations.

Strauss ne change rien aux six premières phrases du monologue, que l'on retrouve au bas du fol. 32v. Le fol. 33r est une page blanche et le fol. 33v une illustration montrant Salomé observant la tête de Jochanaan. Les premières annotations se retrouvent au fol. 34r, transcrit à l'exemple 9. On constate 


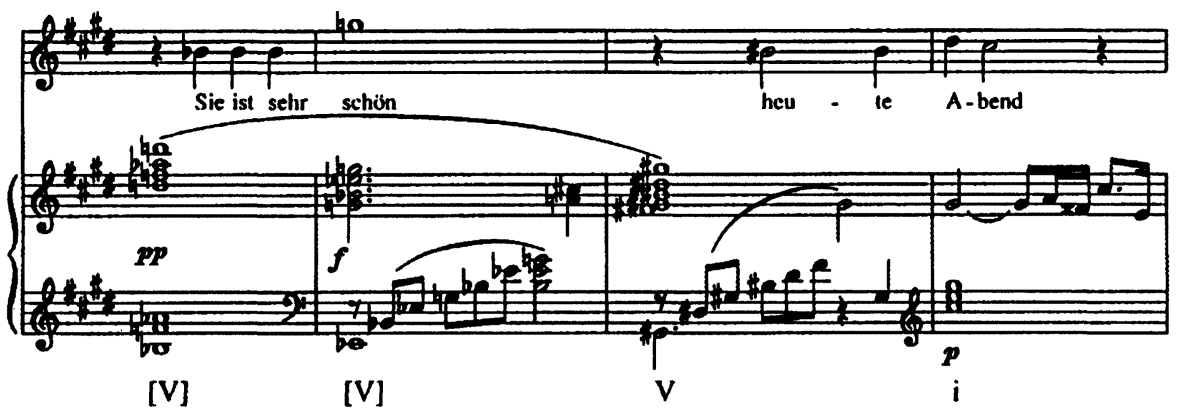

Exemple 6 : Salome, $8^{3}-8^{7}$ (réduction). ( $)$ Copyright 1905 by Adolph Furstner. U.S. copyright renewed. Copyright assigned 1943 to Hawkes \& Son (London) Ltd. (a Boosey \& Hawkes Company) for the world excluding Germany, Italy, Portugal and the former territories of the U.S.S.R. (excluding Estonia, Latvia, and Lithuania).

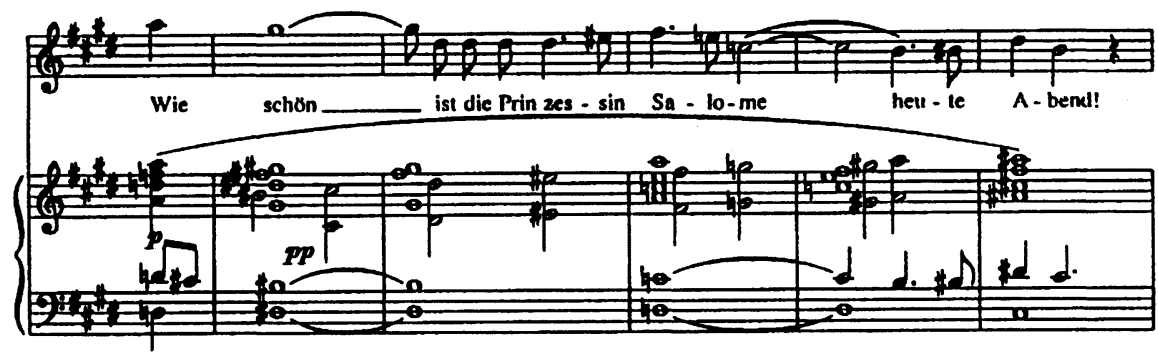

Exemple 7 : Salome, $6^{3}-7^{1}$ (réduction). $\odot$ Copyright 1905 by Adolph Furstner. U.S. copyright renewed. Copyright assigned 1943 to Hawkes \& Son (London) Ltd. (a Boosey \& Hawkes Company) for the world excluding Germany, Italy, Portugal and the former territories of the U.S.S.R. (excluding Estonia, Latvia, and Lithuania).

d'abord que Strauss élimine toutes les allusions à un amour physique de Salomé pour Jochanaan : «Tu n'as pas voulu de moi, Jochanaan. Tu m'as rejetée » (lignes 14-15); " Tu m'as traitée comme une courtisane, comme une prostituée » (lignes 16-17); " tu as été le seul homme que j'aie aimé. Tous les autres hommes m'inspirent du dégoût » (lignes 24-26). On verra que Strauss fera de même dans le reste du monologue, probablement pour conserver jusqu'à la fin l'ambiguité et l'incertitude. La première coupure n'est pas ausi facile à justifier. Peut-être Strauss trouvait-il la sonorité des mots "giftsprühende Schlange » peu propice à la musique.

La page contient aussi trois idées musicales. Celle du haut, marquée « continuo », est une transformation du motif principal de l'opéra dans sa tonalité originale, do dièse mineur. Ce motif en notes courtes est présent dans la partition finale de la mesure $324^{5}$ à la mesure $328^{6}$, c'est-à-dire de « Öffne doch 
DER SKLAVE: Prinzessin, der Tetrarch ersucht $\quad \$$ schnell [20] Euch, wieder zum Fest hineinzugehn. $\left.\begin{array}{l}\text { SALOME: Ich will nicht hineingehn. } \\ \text { PER UNGESYRIER.-Verzeihung, Prinzessin, aber }\end{array}\right\} 3 / 4$ wenn thr nicht hineingeht, kannSehtimmes geschehen. SALOME: Ist dieser Prophet ein alter Mann? DER JUNGE SYRIER: Prinzessin, es wäre besser, — $\$$ hineinzugehn. Gestattet, dass ich Euch führe. SALOME: Ist der Prophet ein alter Mann?

Exemple 8 : Livret annoté, haut du fol. 5 r.

die Augen » (ligne 5) à « Prinzessin von Judäa » (lignes 18-19). Il est présenté successivement dans les tons de sol, de do dièse, de $m i$ bémol et de $s i$ mineur.

La deuxième idée musicale est identifiée «Prinzessin Judäa.» et consiste en un accord de septième de dominante sur $m i$ suivi d'un accord de si bémol mineur, soit un demi-ton plus haut que la résolution normale ${ }^{33}$. Ces deux accords sont conservés par Strauss aux mesures $328^{6-7}$ de l'opéra. La troisième idée musicale, marquée « espr. », semble avoir été destinée à accompagner les mots. « dein Leib war eine Elfenbeinsäule ». Dans sa partition, par contre, Strauss retarde légèrement l'entrée de ce thème et lui fait accompagner les mots "nichts in der Welt war so weiss wie dein Leib », présentés deux phrases plus loin $\left(335^{1-7}\right)$. Les valeurs sont doublées, mais la mélodie et l'harmonie sont inchangées.

L'exemple 10 est une transcription des fol. $34 \mathrm{v}$ et $35 \mathrm{r}$. Les coupures correspondent toutes au texte final de l'opéra, sauf celle du mot "geheimnisvolle » (ligne 8). On voit que Strauss a pensé, à un certain moment, déplacer la phrase « Ich dürste nach deiner Schönheit; ich hungre nach deinem Leib; nicht Wein noch Äpfel können mein Verlangen stillen » pour la mettre à la suite de cette " geheimnisvolle Musik ». Mais il semble s'être ravisé, comme l'indique le mot «bleibt » placé en marge. Dans le texte final, le « $\mathrm{O}$ ! warum » de la ligne 8 devient «Ah! warum », tandis que le «Ah! Ah! warum » de la ligne 26 devient " Oh! warum ». Il est particulièrement intéressant de voir le compositeur couper encore une fois deux passages qui éclairent le comportement de Salomé en en révélant les motivations profondes : «Moi je t'ai vu, Jochanaan, et je t'ai aimé. Je t'aime encore, Jochanaan. Je n'aime que toi » (lignes 16-18) et "J'étais une princesse, tu m'as dédaignée. J'étais une vierge, tu m'as déflorée. J'étais chaste, tu as rempli mes veines de feu » (lignes 23-26). Strauss semble avoir été peu attiré par le mélange d'amour adolescent, de sexualité maladive et d'amoralité qui caractérise chez Wilde le personnage de Salomé.

33Cette technique de transposition chromatique a été observée pour la première fois dans Roland Tenschert, « Die Kadenzbehandlung bei Richard Strauss », Zeitschrift für Musikwissenschaft $8, n^{\circ} 3$ (1925) : 161-83. Cette étude est riche en exemples. 
sagt. Ah! ich will ihn jetzt küssen ... Aber warum siehst du mich nicht an, Jochanaan? Deine Augen, die so schrecklich waren, so voller Wuth und Verachtung, sind jetzt geschlossen. Warum sind sie geschlossen? Öffne doch deine Augen! Erhebe deine Lider, Jochanaan! Warum siehst du mich nicht an? Hast du Angst vor mir, Jochanaan, dass du mich nicht ansehen willst? ... Und deine Zunge, die-wie-eine rothe, giftsprühendefchtange-wor, sie bewegt sichnicht mehr, sie spricht kein Wort, Jochanaan, diese Scharlachnatter, die ihren Geifer auf mich spie. Es ist seltsam, nicht? Wie kommt es, dass die rothe Natter sich nicht mehr rührt? ... Burwolttest michnicht haben, fochaman! Du wiesestmichrondir. Du sprachst böse Worte gegen mich. Bubenahmst dich gegen mich wie gegen eine Hure, wie gegerrein geites Weib, gegen mich, Salome, die Tochter der Herodias, Prinzessin von Judäa! Nun wohl, ich lebe noch, aber du bist tot, und dein Kopf gehört mir. Ich kann mit ihm thun, was ich will. Ich kann ihn den Hunden vorwerfen und den Vögeln der Luft. Was die Hunde übrig lassen, sollen die Vögel der Luft verzehren ... Ah! Jochanaan, Jochanaan, du werrot der Manm, den ich

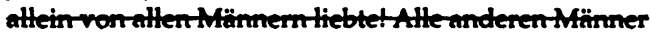
waren mir verhesst. Doch du warst schön! Dein Leib war eine Elfenbeinsäule auf silbernen Füssen.
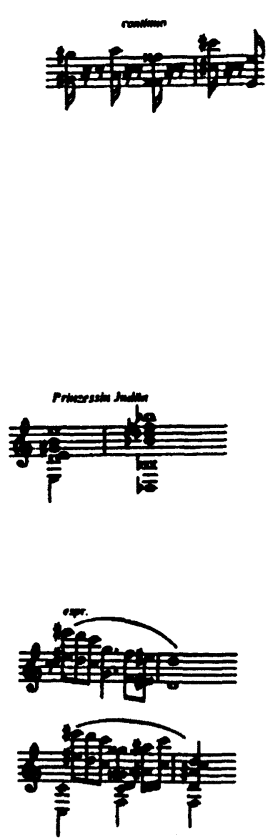

Exemple 9 : Livret annoté, fol. 34 r.

Les annotations des marges du fol. $24 \mathrm{v}$ et celles du fol. $35 \mathrm{r}$, page blanche placée à l'endos d'une illustration, sont de quatre types. On remarquera tout d'abord le numéro de page 73 entre crochets, précédé d'un signe d'insertion. Ce dernier renvoie à la ligne 7 , entre « wenn » et « ich ». C'est là que commence la page 73 de la deuxième édition de la pièce. Deuxièmement, on observe deux indications textuelles pour la composition : la première, "mit Steigerung ", accompagne la troisième notation musicale du fol. $34 \mathrm{v}$, alors que la deuxième, "Wiederholung gesteigert in $\mathrm{X}$ », semble se rapporter à l'idée musicale du bas du fol. 35r. Troisièmement, à la hauteur de la dernière ligne, on retrouve un nom de tonalité accompagné d'une indication de nuance : "Fmoll fo. ». Mais, ce qui frappe le plus dans ces deux pages, c'est sans conteste le grand nombre de notations musicales qu'elles contiennent. La première idée musicale du fol. $34 \mathrm{v}$ (nous l'appellerons idée $A$ ) rappelle le «Ich bin verliebt in deinen Leib » de la première partie de l'opéra. Une flèche indique clairement que Strauss a l'intention d'introduire ce motif pour accompagner les mots "warum hast du mich nicht angesehn ". La deuxième idée du fol. 34v (idée $B$ ) est une progression harmonique ii- $\mathrm{V}^{7}-\mathrm{I}$ en $l a$ bémol majeur. Le $X$ placé au-dessus du système renvoie au $X$ qui précède « Du legtest ». La 


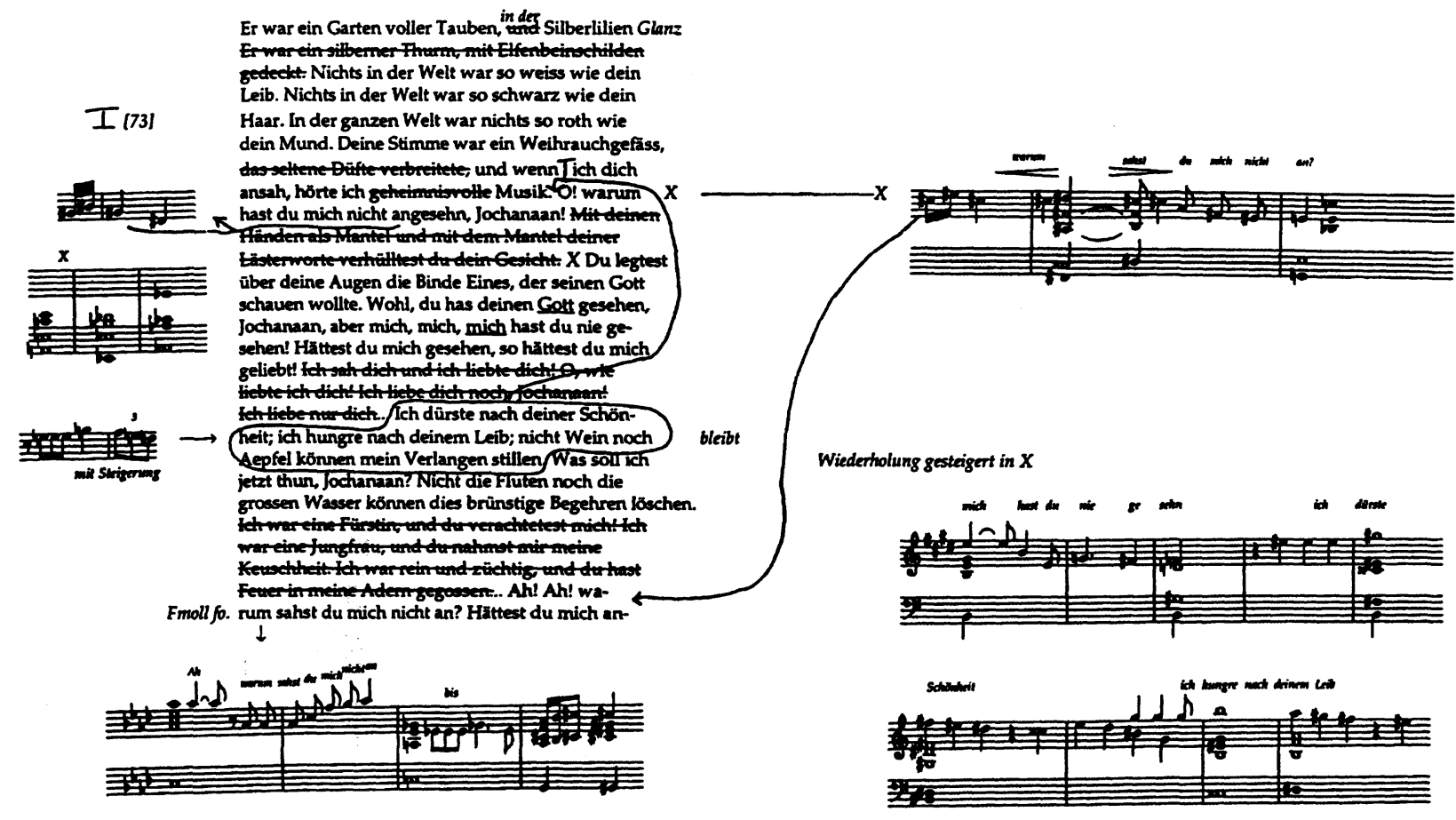

Exemple 10 : Livret annoté, fol. $34 \mathrm{v}$ et $35 \mathrm{r}$. 
troisième idée musicale (idée $C$ ) - un motif associé, dans la première partie de l'opéra, à « ich will deinen Mund küssen »- est clairement reliée par Strauss au passage encerclé. Le passage noté au bas du fol. 34v (idée $D$ ) doit accompagner les mots " warum sahst du mich nicht an ». On y remarquera le motif « Ich will den Kopf ». Le fol. 35r contient deux passages. Le premier (idée $E$ ) met en musique le même texte que l'extrait musical du bas du fol. 34v. Mais on remarquera également le $X$ placé à sa gauche, qui semble renvoyer au $X$ placé à droite de la ligne 8 du texte. Finalement, l'extrait du bas (idée $F$ ), qui est aussi le plus long, est clairement destiné au passage encerclé.

Penchons-nous maintenant sur chacune de ces idées musicales et tentons d'établir leur degré de parenté avec le texte musical final de l'opéra. L'idée $A$ n'est pas employée en sol dièse ou en la bémol majeur dans la version finale de l'opéra. Par contre, comme on peut le voir à l'exemple 11, le « Ah! warum hast du mich nicht angesehn » y est accompagné par une musique clairement dérivée de l'idée $E$. Les valeurs rythmiques sont pour la plupart doublées, mais l'harmonie de base reste la même. On peut donc maintenant tenter de reconstituer hypothétiquement le processus compositionnel : Strauss décide d'abord d'utiliser le motif de l'idée $A$ en sol dièse minèur. Puis, pour accompagner les deux dernières lignes de texte, il note l'idée $E$. Mais le début de celle-ci lui semble trop près de l'idée $A:$ il juge que ce motif ne devrait pas revenir aussi rapidement. Il décide donc d'utiliser l'idée $E$ à la place de l'idée $A$, d'où les $X$ mentionnés plus haut.

L'idée $B$ n'est qu'un schéma harmonique. Une comparaison avec le résultat final nous montre à quel point les premières idées musicales de Strauss, bien que souvent très simples, peuvent donner naissance à des passages beaucoup plus complexes. Ici, Strauss étend l'action des deux premiers accords sur huit mesures $\left(340^{8}-41^{4}\right)$. Puis, il transpose encore une fois l'accord de résolution d'un demi-ton vers le bas pour obtenir l'accord de sol mineur $\left(341^{5}\right)$. Le passage est reproduit à l'exemple 12.

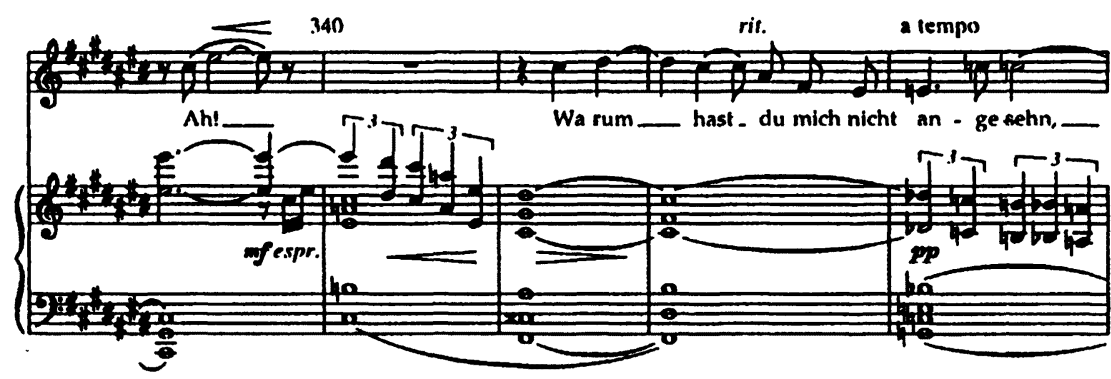

Exemple 11 : Salome, $339^{6}-40^{4}$ (réduction). (C) Copyright 1905 by Adolph Furstner. U.S. copyright renewed. Copyright assigned 1943 to Hawkes \& Son (London) Ltd. (a Boosey \& Hawkes Company) for the world excluding Germany, Italy, Portugal and the former territories of the U.S.S.R. (excluding Estonia, Latvia, and Lithuania). 

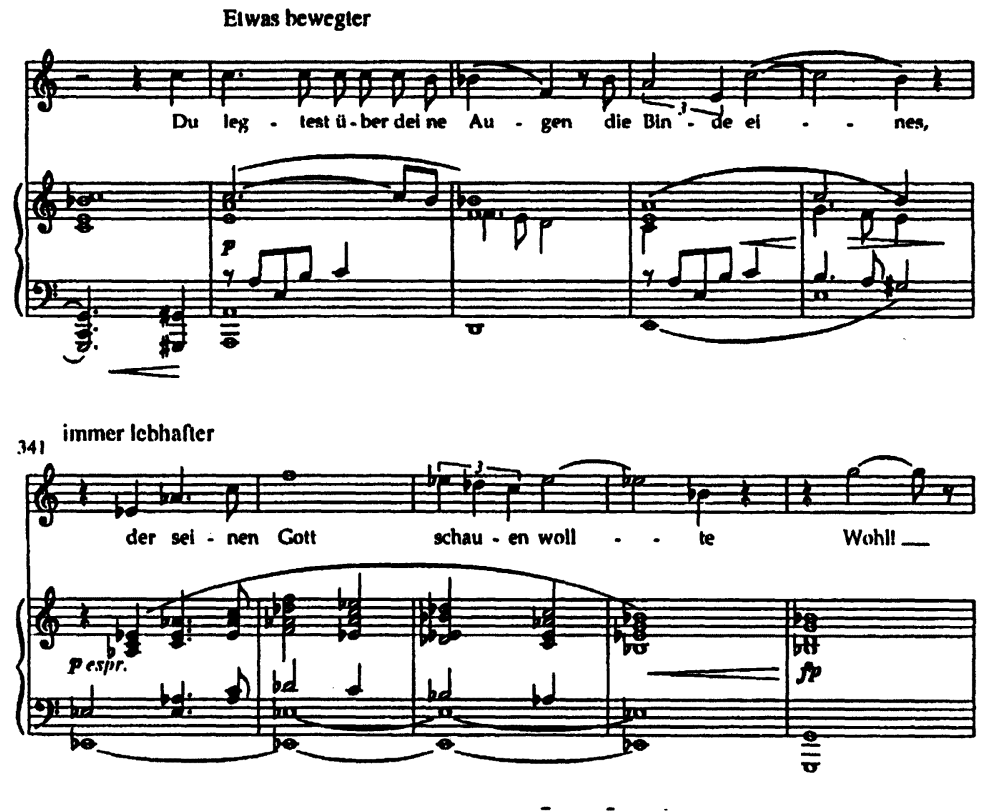

Exemple 12 : Salome, $340^{7}-41^{5}$ (réduction). @ Copyright 1905 by Adolph Furstner. U.S. copyright renewed. Copyright assigned 1943 to Hawkes \& Son (London) Ltd. (a Boosey \& Hawkes Company) for the world excluding Germany, Italy, Portugal and the former territories of the U.S.S.R. (excluding Estonia, Latvia, and Lithuania).

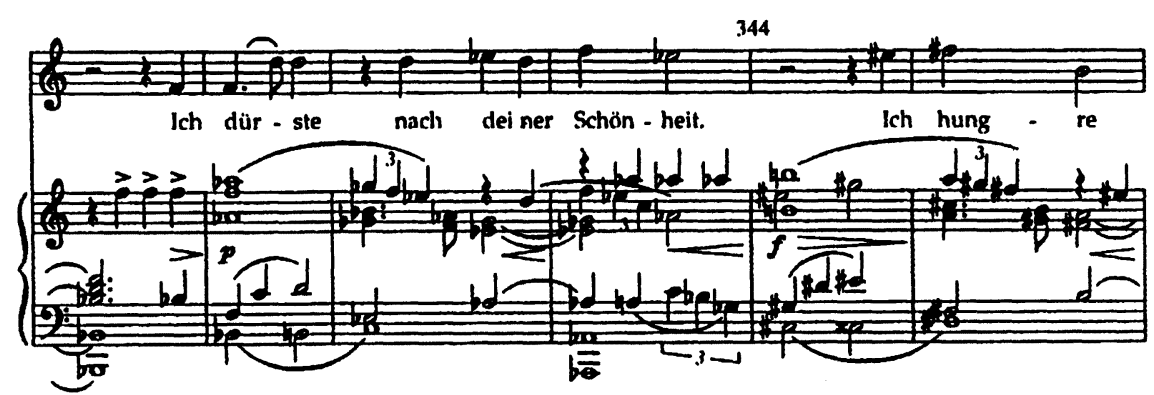

Exemple 13 : Salome, $343^{3}-44^{2}$ (réduction). $\odot$ Copyright 1905 by Adolph Furstner. U.S. copyright renewed. Copyright assigned 1943 to Hawkes \& Son (London) Ltd. (a Boosey \& Hawkes Company) for the world excluding Germany, Italy, Portugal and the former territories of the U.S.S.R. (excluding Estonia, Latvia, and Lithuania). 

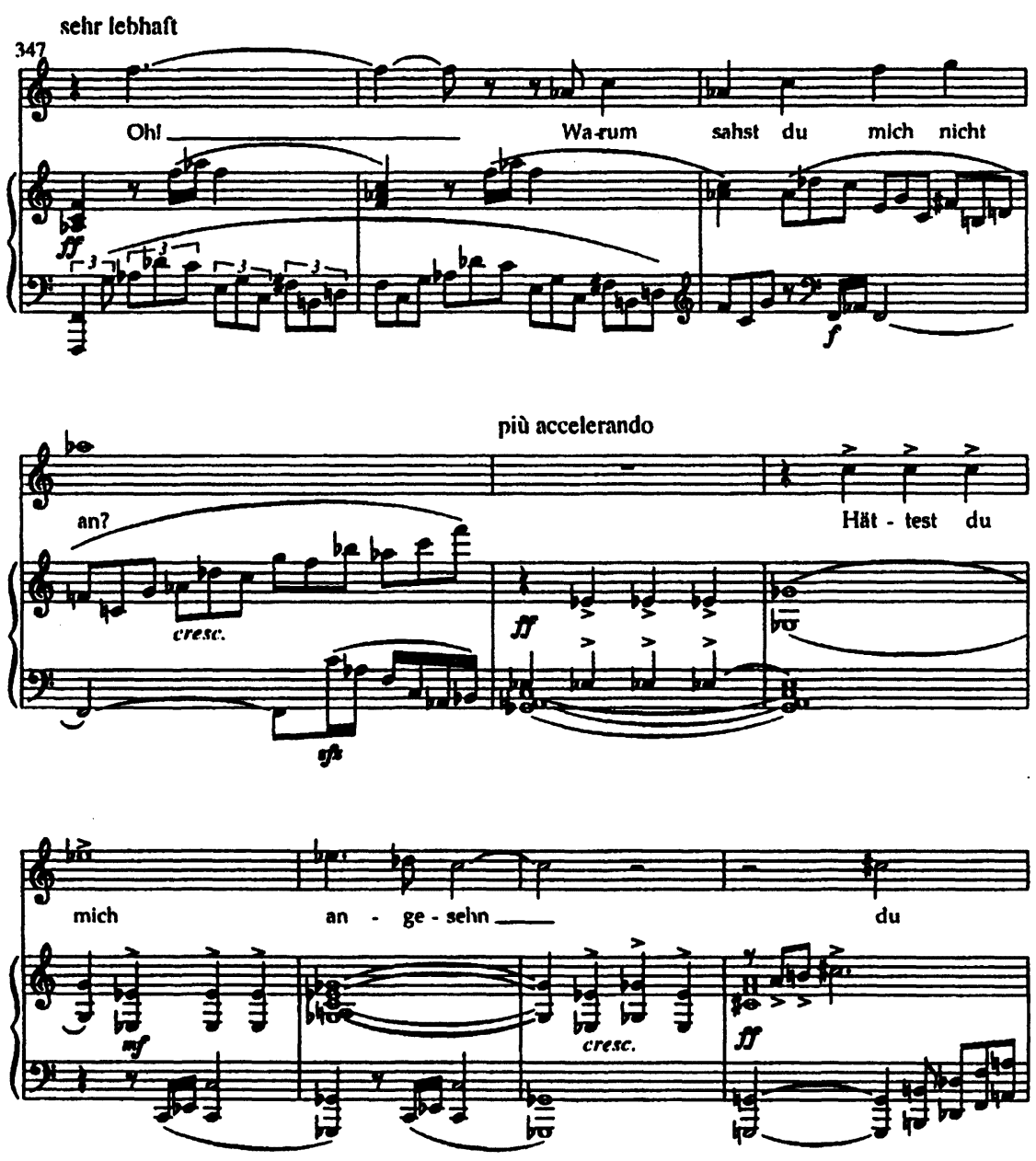

Exemple 14 : Salome, $347^{1}-47^{10}$ (réduction). (C Copyright 1905 by Adolph Furstner. U.S. copyright renewed. Copyright assigned 1943 to Hawkes \& Son (London) Ltd. (a Boosey \& Hawkes Company) for the world excluding Germany, Italy, Portugal and the former territories of the U.S.S.R. (excluding Estonia, Latvia, and Lithuania).

Les idées $C$ et $F$ sont toutes deux reliées au passage encerclé. Strauss semble avoir d'abord noté $C$, puis s'être servi de ce motif pour écrire $F$. Si on compare $F$ avec le résultat final $\left(342^{3}-44^{4}\right)$, on constate que Strauss a oublié une phrase dans son annotation ( « Hättest du mich gesehn, du hättest mich geliebt! ») et qu'il a donc dû transformer complètement les trois premières mesures. Par contre, comme on peut le vérifier à l'exemple 13, le reste de $F$ a bien été utilisé dans l'opéra. On remarquera encore une fois l'utilisation d'une notation enharmonique.

L'idée $D$, marquée « Fmoll fo. », sert de formule génératrice à 10 mesures de la partition finale. Ces 10 mesures, de $347^{1}$ à $347^{10}$, sont reproduites à 
l'exemple 14. On reconnaît aisément les transformations effectuées : premièrement, les deux premières mesures de $D$ deviennent les quatre premières mesures de l'exemple à cause d'une augmentation des valeurs; deuxièmement, la troisième mesure de $D$, marquée « bis ", génère cinq mesures par doublement des valeurs et ajout d'une mesure $\left(347^{9}\right)$; troisièmement, la quatrième mesure de $D$ devient la mesure $347^{10}$ et Strauss retarde donc l'accord de do dièse majeur en deuxième renversement, qui se trouve à la mesure $348^{1}$.

Le monologue se termine au haut du fol. 36 r, car, comme nous l'avons mentionné plus haut, le fol. 35v est une page d'illustration. Ce court passage ne contient que deux indications de tonalités : "Cisdur » au-dessus de « hättest mich » et « Esmoll » à la fin de la dernière ligne.

Ces quelques exemples nous ont permis de constater à quel point la sonorité générale de l'opéra et un bon nombre d'idées thématiques et harmoniques prennent forme dans l'esprit de Strauss dès le travail sur le livret. On a aussi pu constater sa propension à associer certains mots et certaines images à des accords ou à des tonalités fixes. Bien entendu, ces gloses marginales, ces coupures, ces transformations du texte, ces planifications rythmiques, ne résisteront pas toutes, on l'a vu, au travail ultérieur de perfectionnement. Il en restera tout de même une certaine direction générale, un matériau essentiel. Ce sont ces éléments, qui n'avaient encore jamais été identifiés et classés, que devront retenir les analystes et les interprètes soucieux de séparer le fondamental du décoratif.

\section{Résumé}

L'auteur, après avoir tenté d'établir à quel moment Richard Strauss commence son travail sur Salome, fait un inventaire des sources manuscrites de l'opéra. Puis il se concentre sur un exemplaire de la première édition de la traduction allemande de la pièce d'Oscar Wilde ayant appartenu au compositeur et qui contient de nombreuses annotations musicales. Une classification et une description de celles-ci sont suivies de l'analyse détaillée du premier stade de conception de deux passages clés de Salome. 